\title{
Impaired Cognitive Function Due to Cerebellar Infarction and Improvement After Stent-Assisted Angioplasty for Intracranial Vertebral Artery Stenosis
}

\author{
-Case Report-
}

\author{
Yoshiro Ito, Yuji MAtsumaru*, Kensuke SuZUKI, and Akira MATSUmURA \\ Department of Neurosurgery, Institute of Clinical Medicine, \\ University of Tsukuba, Tsukuba, Ibaraki; \\ ${ }^{*}$ Department of Interventional Neuroradiology, Toranomon Hospital, Tokyo
}

\begin{abstract}
A 69-year-old female presented with multiple cerebellar infarctions and mild cognitive dysfunction. Cerebral angiography revealed severe right vertebral artery (VA) stenosis just proximal to the origin of the posterior inferior cerebellar artery. Neuropsychology tests showed the mini-mental state examination (MMSE) score was 21, and the Wechsler Adult Intelligence Scale-Revised (WAIS-R) score was 70. Cerebrovascular reactivity (CVR) was decreased in the bilateral cerebral and cerebellar hemispheres. Stent-assisted angioplasty of the right VA was performed to prevent recurrence of the cerebellar infarction. Post-procedure, the neuropsychology tests showed cognitive improvement to MMSE score of 26 and WAIS-R score of 84. CVR had also improved in the bilateral cerebral hemispheres. Cerebellar hypoperfusion due to VA stenosis had probably caused functional depression of the cerebello-cerebral pathway, a condition referred to as crossed cerebello-cerebral diaschisis. In this case, cognitive dysfunction was reversed due to recovery of the cerebello-cerebral pathway.
\end{abstract}

Key words: vertebral artery stenosis, stent-assisted angioplasty, cognitive dysfunction, crossed cerebello-cerebral diaschisis, cerebello-cerebral pathway

\section{Introduction}

Impairment of the unilateral cerebellar hemisphere inhibits perfusion of the contralateral cerebral hemisphere due to dysfunction of the cerebello-cerebral pathway. ${ }^{2)}$ This phenomenon is called crossed cerebello-cerebral diaschisis (CCCD), and may affect cognitive function. ${ }^{1,10}$ ) CCCD might be explained by functional depression of the cerebello-cerebral pathway that is dependent on a reduction in cerebral blood flow (CBF). ${ }^{2,6,13)}$ Therefore, cerebellar lesions, especially in the posterior regions of the cerebellum fed by the posterior inferior cerebellar artery (PICA), may lead to the deterioration of cognitive function and affective regulation. . $^{3,4,7,10)}$

We treated a patient with vertebral artery (VA) stenosis by stent-assisted angioplasty, followed by improvement in cerebrovascular reactivity (CVR) and reversal of cognitive dysfunction, which illustrates the relationship between cognitive dysfunction and reperfusion of the cerebellar circulation.

\section{Case Report}

A 69-year-old female consulted a physician for complaints of vertigo, dysarthria, and gait disturbance. Mild memory disturbance had been detected by the patient's family. Magnetic resonance (MR) imaging of the brain showed multiple cerebellar infarctions at a local hospital. She was transferred to our hospital for further examinations and treatment.

The patient had a past history of hypertension and antihypertensive drug therapy. She did not drink alcohol and never smoked. She was right-handed. Her academic background was junior high school graduate. She became a housewife after marriage. Neurological examination on admission disclosed dysarthria and ataxic gait, both caused by cerebellar disorder. The patient received continuous treatment with intravenous aspirin (100 mg/dl) and ozagrel $(160 \mathrm{mg} / \mathrm{dl})$. The mini-mental state examination (MMSE) showed a score of 21 out of 30. The Wechsler Adult Intelligence Scale-Revised (WAIS-R) showed a score of 70; verbal intelligence quotient (IQ) was 82 and performance IQ was 58. Neuropsychological examinations rev-

Received March 16, 2009; Accepted July 15, 2009

Author's present address: Y. Ito, M.D., Department of Neurosurgery, Mito Medical Center, Higashi Ibaraki-gun, Ibaraki, Japan. 

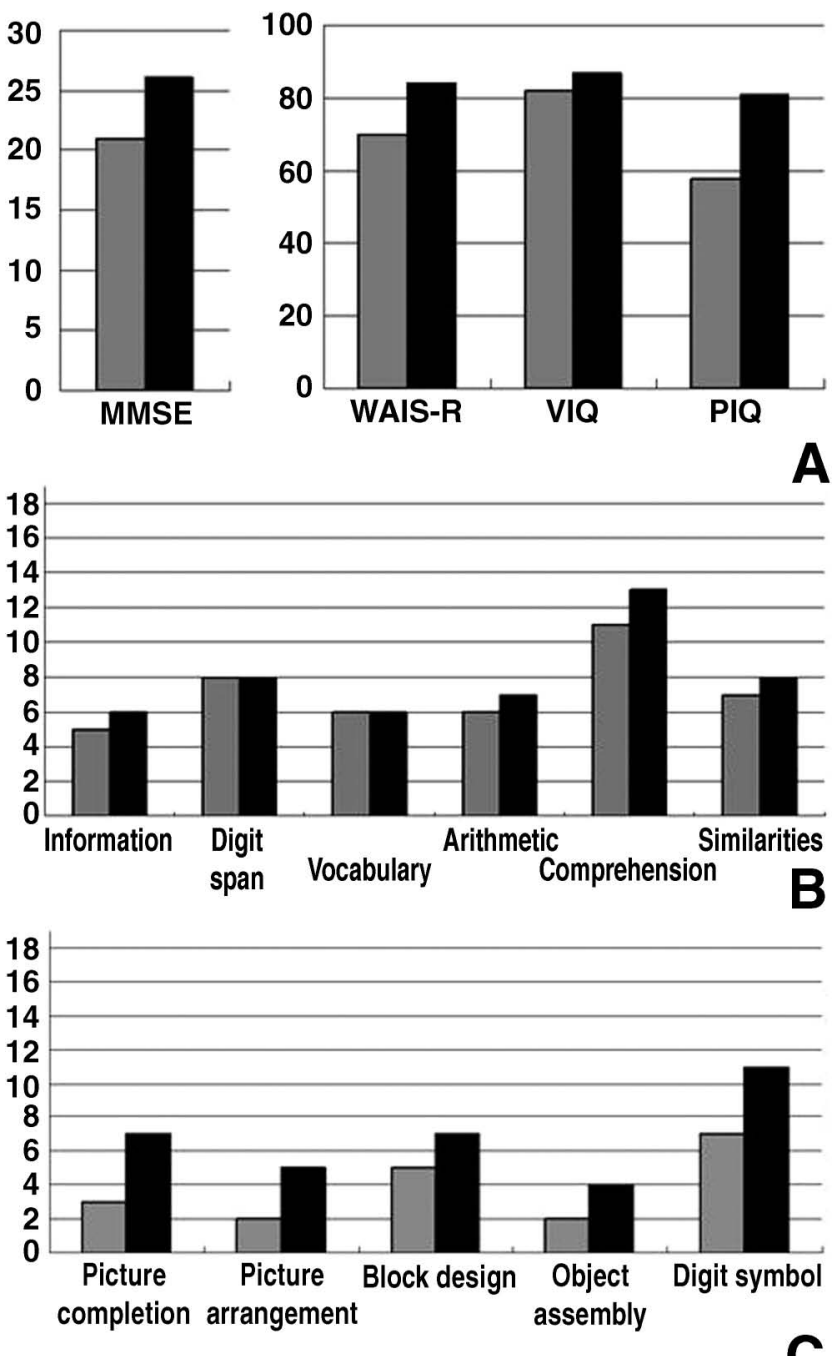

Fig. 1 Results of the pre- (shaded columns) and post-procedure (black columns) neuropsychology tests. A: Both the mini-mental state examination (MMSE) and the Wechsler Adult Intelligence Scale-Revised (WAIS-R) scores had improved, especially the performance intelligence quotient (PIQ). VIQ: verbal intelligence quotient. B: Scaled scores of verbal subsets. C: Scaled scores of performance subsets.
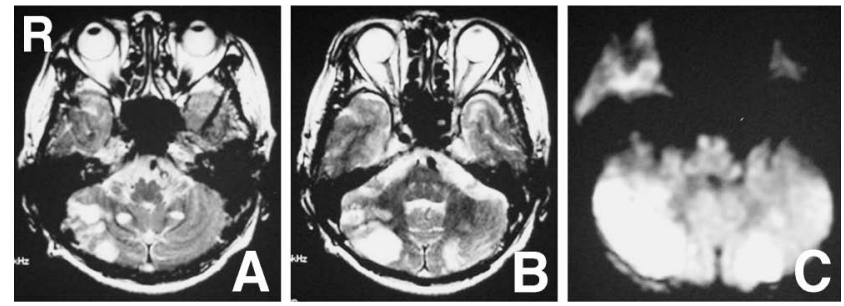

Fig. 2 A, B: $T_{2}$-weighted magnetic resonance images on admission showing multiple old cerebellar infarctions. C: Diffusion-weighted image showing multiple fresh cerebellar infarctions.
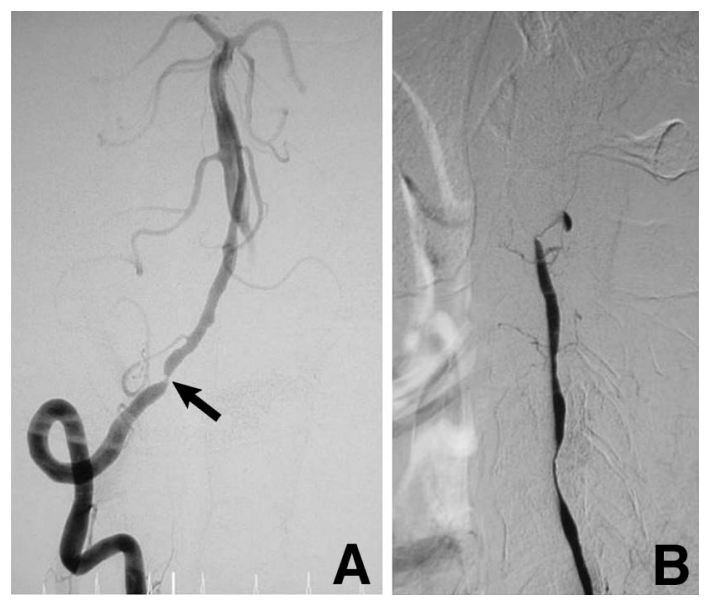

Fig. 3 A: Right vertebral angiogram, anteroposterior view, revealing severe stenosis of the right vertebral artery just proximal to the origin of the posterior inferior cerebellar artery (arrow). B: Left vertebral angiogram, lateral view, revealing occlusion of the left vertebral artery.

ealed mild cognitive dysfunction (Fig. 1).

MR imaging revealed new as well as old infarctions in the PICA area of the bilateral cerebellum (Fig. 2). Cerebral angiography revealed severe stenosis of the right VA just proximal to the origin of the PICA, and occlusion of the left VA (Fig. 3). No collateral flow to the posterior circulation was seen. Single photon emission computed tomography (SPECT) using technetium-99m ethyl cysteinate dimer showed no deterioration of the CBF, but CVR was reduced across the entire brain, remarkably in the cerebellum (Fig. 4). CBF was $43.3 \mathrm{ml} / 100 \mathrm{~g} / \mathrm{min}$ in the right cerebellar hemisphere and $42.1 \mathrm{ml} / 100 \mathrm{~g} / \mathrm{min}$ in the left cerebellar hemisphere. The CVR to acetazolamide was $3.5 \%$ in the right cerebellar hemisphere and $4.3 \%$ in the left cerebellar hemisphere (Table 1). We concluded that the patient was at high risk of recurrence of cerebellar infarction, as the left VA was occluded and the right VA showed severe stenosis. Consequently, stent-assisted angioplasty for the right VA was scheduled.

All procedures were performed with the patient under general anesthesia. After placement of a 7-French sheath in the right femoral artery, a bolus dose of heparin (100 $\mathrm{U} / \mathrm{kg}$ ) was administered intravenously to obtain an activated clotting time of approximately 300 seconds. A 6French, straight, Envoy guiding catheter (Cordis Endovascular, Miami Lakes, Fla., U.S.A.) was selectively placed in the right VA. A Gateway 2.5- $\times 12-\mathrm{mm}$ balloon (Boston Scientific Japan, Tokyo) was guided over the wire and inflated at $6 \mathrm{~atm}$. After predilation, an S670 3- $\times$ 9-mm stent mounted on its delivery balloon (Boston Scientific Japan) was advanced over the wire and positioned across the lesion. The balloon was inflated at $7 \mathrm{~atm}$. and the stent was deployed. Postdilation was performed with the Gateway $2.5-\times 12-\mathrm{mm}$ balloon inflated at $14 \mathrm{~atm}$. Cerebral angiography after the procedure demonstrated amelioration of the VA stenosis (Fig. 5A). Continuous infusion of heparin was administered intravenously for 24 hours after 

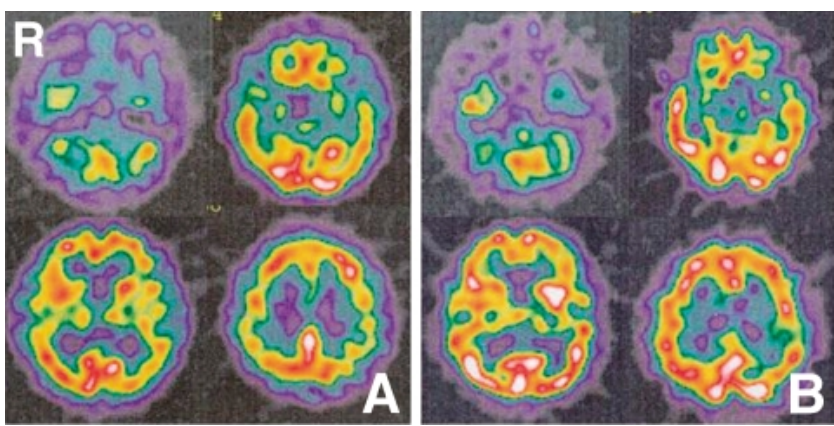

Fig. 4 Single photon emission computed tomography scans using technetium-99m ethyl cysteinate dimer, at rest (A) and after acetazolamide stress test (B), revealing no deterioration in cerebral blood flow, but reduced cerebrovascular reactivity across the entire brain, especially in the bilateral cerebellar hemispheres.

Table 1 Cerebral blood flow (CBF) and cerebrovascular reactivity (CVR) studies by single photon emission computed tomography

\begin{tabular}{lccrrr}
\hline & \multicolumn{2}{c}{ CBF $(\mathrm{ml} / 100 \mathrm{~g} / \mathrm{min})^{*}$} & & \multicolumn{2}{c}{ CVR (\%)* } \\
\cline { 2 - 3 } \cline { 5 - 6 } & Right & Left & & Right & \multicolumn{1}{c}{ Left } \\
\hline Frontal & $41.2 / 44.3$ & $43.1 / 43.2$ & & $15.5 / 16.3$ & $6.0 / 23.8$ \\
Parietal & $41.8 / 43.0$ & $40.4 / 40.1$ & & $20.8 / 23.3$ & $11.6 / 25.7$ \\
Temporal & $38.8 / 39.6$ & $36.2 / 36.1$ & & $14.4 / 19.7$ & $13.5 / 26.6$ \\
Occipital & $48.7 / 49.3$ & $46.8 / 47.1$ & & $8.4 / 25.4$ & $12.0 / 28.5$ \\
Thalamus & $38.0 / 42.4$ & $41.2 / 38.6$ & & $9.7 / 30.9$ & $4.4 / 50.8$ \\
Cerebellum & $43.3 / 44.3$ & $42.1 / 40.5$ & & $3.5 / 24.6$ & $4.3 / 29.9$ \\
\hline
\end{tabular}

*Values are before procedure/at 6-month follow up.
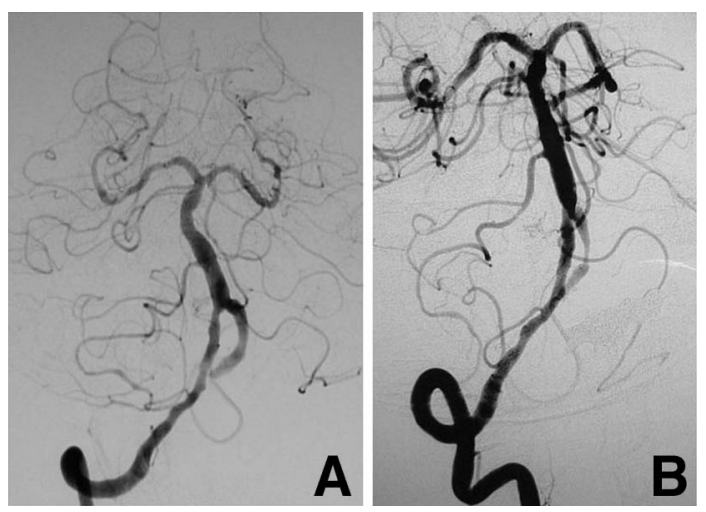

Fig. 5 A: Post-procedure right vertebral angiogram demonstrating improvement of the stenosis of the right vertebral artery. B: Right vertebral angiogram at 4-month follow-up examination revealing no significant restenosis.

procedure. The presenting symptoms were ameliorated by rehabilitation. The patient was discharged without assistance, and without complications or neurological events.

Cerebral angiography at the 4-month follow-up examination revealed no significant restenosis (Fig. 5B). SPECT
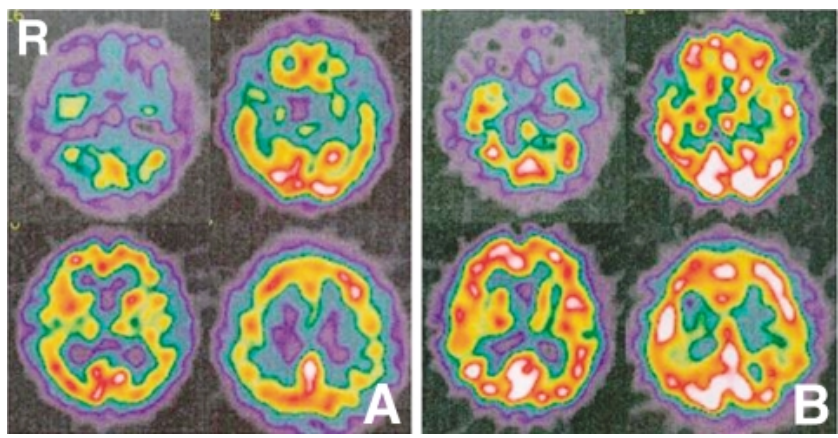

Fig. 6 Single photon emission computed tomography scans using technetium $-99 \mathrm{~m}$ ethyl cysteinate dimer, at rest $(A)$ and after acetazolamide stress test (B), at 6-month follow-up examination showing that cerebrovascular reactivity in the bilateral cerebral and cerebellar hemispheres had increased to levels higher than those observed prior to the procedure.

at a 6-month follow-up examination showed that CVR to acetazolamide in the bilateral cerebral and cerebellar hemispheres had increased to levels higher than those observed prior to the procedure (Fig. 6). CBF was 44.3 $\mathrm{ml} / 100 \mathrm{~g} / \mathrm{min}$ in the right cerebellar hemisphere and 40.5 $\mathrm{ml} / 100 \mathrm{~g} / \mathrm{min}$ in the left cerebellar hemisphere. The CVR to acetazolamide was $24.6 \%$ in the right cerebellar hemisphere and $29.9 \%$ in the left cerebellar hemisphere (Table 1).

Eighteen months after the procedure, the patient still exhibited no neurological symptoms, i.e., no gait disturbance, no nystagmus, and fluent speech. The MMSE score had improved to 26 out of 30 . The WAIS-R results had also improved to a score of 84 ; verbal IQ was 87 and performance IQ was 81 (Fig. 1). The patient continued to receive two antiplatelet drugs, aspirin and ticlopidine.

\section{Discussion}

Stent-assisted angioplasty for symptomatic carotid artery stenosis is a therapeutic technique that can enhance cerebral perfusion, which in turn may reverse cognitive dysfunction. ${ }^{8,11,12)}$ However, only a few cases have been reported in which stent-assisted angioplasty for VA stenosis improved cerebral hypoperfusion and cognitive dysfunction. ${ }^{9,11)}$ Unfortunately, neither of these reports addressed the pathophysiology of cerebral perfusion, or described any cognitive dysfunction. In the present case, the cognitive dysfunction was apparently associated with CCCD, as shown by the simultaneous improvement with recovery of the cerebello-cerebral pathway after stent-assisted angioplasty for the VA occlusion, which increased CVR in both the cerebellum and the cerebrum. Furthermore, the performance IQ was especially improved after the angioplasty. Performance IQ is said to be associated with the right cerebral hemisphere. Angioplasty for the right VA occlusion recovered the perfusion of the left PICA areas, so presumably CBF of the left cerebellar hemisphere was increased and CCCD was improved. However, the transition of the cerebellar ataxia led to the 
improvement of performance IQ.

The cerebellum may also be associated with cognitive function. The cerebellum is suggested to modulate both the motor system and cognitive function. ${ }^{4)}$ Cerebellar cognitive function shows some localization. Lesions of the PICA areas result in cognitive and affective deficits, which suggests that the posterior cerebellar regions are dominant in cognitive and affective processing. ${ }^{3}$ Clinically significant cognitive impairments develop in the posteroinferolateral and posteromedial areas of the cerebellar cortex associated with the dominant hemisphere of the brain. ${ }^{5)}$ The cerebellum is also involved in the processing of information arriving from the motor zones of the cerebral cortex, and from areas associated with cognitive function. ${ }^{5)}$ The PICA areas of the cerebellum may also possess cognitive function. In the present case, any direct effect of the stent-assisted angioplasty on the cognitive function of the cerebellum is difficult to establish because no changes were observed in the CBF of the cerebellum.

The present case of severe right VA stenosis just proximal to the origin of the PICA with multiple cerebellar infarctions was associated with cognitive dysfunction, which was ameliorated after stent-assisted angioplasty for the VA stenosis. The cognitive dysfunction may have been associated with CCCD. Angioplasty for improving the posterior circulation may also improve cognitive function in patients with CCCD.

\section{References}

1) Boni S, Valle G, Cioffi RP, Bonetti MG, Perrone E, Tofani A, Maini CL: Crossed cerebello-cerebral diaschisis: a SPECT study. Nucl Med Commun 13: 824-831, 1992

2) Broich K, Hartmann A, Biersack HJ, Horn R: Crossed cerebello-cerebral diaschisis in a patient with cerebellar infarction. Neurosci Lett 83: 7-12, 1987

3) Exner C, Weniger G, Irle E: Cerebellar lesions in the PICA but not SCA territory impair cognition. Neurology 63: 2132-2135, 2004
4) Gasparini M, Piero VD, Ciccarelli O, Caccioppo MM, Pantano P, Lenzi GL: Linguistic impairment after right cerebellar stroke: a case report. Eur J Neurol 6: 353-356, 1999

5) Kalashnikova LA, Zueva YV, Pugacheva OV, Korsakova NK: Cognitive impairments in cerebellar infarcts. Neurosci Behav Physiol 35: 773-779, 2005

6) Komaba Y, Osono E, Kitamura S, Katayama Y: Crossed cerebellocerebral diaschisis in patients with cerebellar stroke. Acta Neurol Scand 101: 8-12, 2000

7) Leiner H, Leiner A, Dow R: The human cerebro-cerebellar system: its computing, cognitive, and language skills. Behav Brain Res 44: 113-128, 1991

8) Levy EI, Horowitz MB, Koebbe CJ, Jungreis CC, Pride GL, Dutton K, Purdy PD: Transluminal stent-assisted angioplasty of the intracranial vertebrobasilar system for medically refractory, posterior circulation ischemia: Early results. Neurosurgery 48: 1215-1223, 2001

9) Maeshima $S$, Terada $T$, Yoshida $N$, Nakai $K$, Itakura $T$, Komai N: Cerebral angioplasty in a patient with vascular dementia. Arch Phys Med Rehabil 78: 666-669, 1997

10) Marien P, Engelborghs S, Fabbro F, De Deyn PP: The lateralized linguistic cerebellum: a review and a new hypothesis. Brain Lang 79: 580-600, 2001

11) Moftakhar R, Turk AS, Niemann DB, Hussain S, Rajpal S, Cook T, Geraghty M, Aagaard-Kienitz B, Turski PA, Newman GC: Effects of carotid or vertebrobasilar stent placement on cerebral perfusion and cognition. AJNR Am J Neuroradiol 26: 1772-1780, 2005

12) Rasmussen PA, Perl JII, Barr JD, Markarian GZ, Katzan I, Sila C, Krieger D, Furlan AJ, Masaryk TJ: Stent-assisted angioplasty of intracranial vertebrobasilar atherosclerosis: an initial experience. J Neurosurg 92: 771-778, 2000

13) Rousseaux M, Steinling M: Crossed hemispheric diaschisis in unilateral cerebellar lesions. Stroke 23: 511-514, 1992

Address reprint requests to: Yoshiro Ito, M.D., Department of Neurosurgery, Mito Medical Center, 280 Sakuranosato, Ibaraki-machi, Higashi Ibaraki-gun, Ibaraki 311-3193, Japan.

e-mail: Yoshiro@apr.email.ne.jp 